\title{
POTRILLO, NUEVO HÍBRIDO DE SORGO DULCE PARA LA REGIÓN CENTRAL DE NUEVO LEÓN Y SUR DE TAMAULIPAS, MÉXICO
}

\section{POTRILLO, A NEW SWEET SORGHUM HYBRID FOR CENTRAL NUEVO LEON AND SOUTHERN TAMAULIPAS, MÉXICO}

\author{
Héctor Williams-Alanís ${ }^{1 *}$, Francisco Zavala-García², Gerardo Arcos-Cavazos ${ }^{3}$, \\ Ma. del Carmen Rodríguez-Vázquez², Emilio Olivares-Sáenz ${ }^{2}$ y J. Elías Treviño-Ramírez ${ }^{2}$
}

\begin{abstract}
'Instituto Nacional de Investigaciones Forestales, Agrícolas y Pecuarias (INIFAP), Campo Experimental Río Bravo, Río Bravo, Tamaulipas, México. 2Universidad Autónoma de Nuevo León, Facultad de Agronomía, Marín, Nuevo León, México. ${ }^{3}$ INIFAP, Campo Experimental Huastecas, Estación Cuauhtémoc, Tamaulipas, México.
\end{abstract}

*Autor de correspondencia (hectorwilliamsa@yahoo.com.mx)

El sorgo dulce [Sorghum bicolor L. (Moench)] es un cultivo con alto potencial para producir bioetanol. En México, la Secretaría de Agricultura y Desarrollo Rural (SADER) y el Consejo Nacional de Ciencia y Tecnología (CONACYT) impulsan el desarrollo de nuevas variedades de sorgo dulce con mayor potencial de producción.

Potrillo es un híbrido de sorgo dulce resultado de la cruza de Potranca $\times$ Keller. Potranca es una línea androestéril que se generó en la Universidad Autónoma de Nuevo León (UANL) durante los años 2008 a 2012 (Williams-Alanís et al., 2017). El progenitor donante de la androesterilidad fue la línea granífera 46038, desarrollada por la Universidad de Nebraska, EE.UU. La línea mantenedora es Rox Orange (o Waconia), que es una variedad de sorgo dulce y forrajera que se produjo por la Estación Agrícola Experimental de Wisconsin, EE.UU. (Undersander et al., 1990). Keller, progenitor masculino de Potrillo, es una variedad de sorgo dulce desarrollada por Sugar Crops Field Station en Meridian, Mississippi, EE.UU. a partir de Rio (Murray et al., 2009). El registro de Potrillo a la fecha se encuentra en trámite ante el SNICS (Expediente 3503), lo mismo que el Título de Obtentor (Expediente 2855).

En el año 2012 se evaluaron cinco híbridos experimentales, entre los que destacó la cruza Potranca $\times$ Keller (Potrillo), la cual posteriormente se evaluó en seis ambientes del noreste de México: Estación Cuauhtémoc en secano, en los ciclos agrícolas de verano 2013, otoño-invierno 2014 y verano 2014, y en Marín (UANL) en riego, durante la primavera 2014, verano 2014 y verano 2015.

El nuevo híbrido de sorgo dulce Potrillo presenta granos pequeños, color marrón rojizo y panojas semicompactas (Figura 1). Su rendimiento promedio de biomasa total es de 49,131 kg ha-1; distribuida en tallos: 37,243; hojas: 7467 y jugo: 13,361 kg ha-1 (Cuadro 1), con lo que supera al mejor testigo (Keller) en 25.9, 23.5, 36.4 y $26.6 \%$, respectivamente. Potrillo presenta $14.8{ }^{\circ}$ Brix, valor menor que el de los testigos Keller (16.2 ${ }^{\circ}$ Brix) y Urja (16.4 ${ }^{\circ}$ Brix), pero mayor que RB-Cañero (9.4 ${ }^{\circ}$ Brix). La producción de bioetanol se estima en 2026 L ha-1 para Potrillo, 1640 para Keller, 1780 para Urja y 1316 para RB-Cañero, lo que equivale a 23.5, 11.4 y $54 \%$ de superioridad, respectivamente. Potrillo es más precoz y más tolerante al acame que Keller y RBCañero, aunque de porte similar (Cuadro 1).

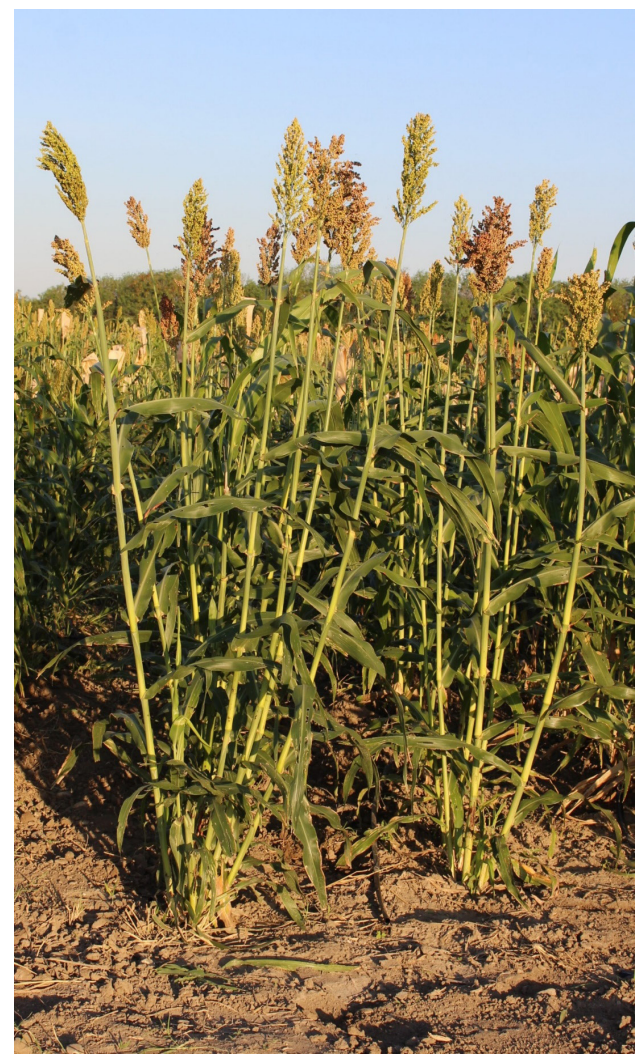

Figura 1. Aspecto general de las plantas del híbrido de sorgo dulce Potrillo 
Cuadro 1. Medias de características agronómicas de Potrillo y testigos en seis experimentos durante los años 2013, 2014 y 2015. Marín, N. L. y Estación Cuauhtémoc, Tam.

\begin{tabular}{|c|c|c|c|c|c|}
\hline Variedad & Biomasa $\left(\mathrm{kg} \mathrm{ha}^{-1}\right)$ & Tallo $\left(\mathrm{kg} \mathrm{ha}^{-1}\right)$ & Hoja $\left(\mathrm{kg} \mathrm{ha}^{-1}\right)$ & Jugo (kg ha-1) & ${ }^{\circ}$ Brix \\
\hline Potrillo & 49,131 a & $37,243 a$ & 7467 a & 13,361 a & $14.8 \mathrm{~b}$ \\
\hline Keller $(T)$ & $39,025 b$ & $30,145 b$ & $5473 a b$ & $10,556 \mathrm{bc}$ & $16.2 a b$ \\
\hline Urja $(T)$ & $35,413 b$ & $32,734 a b$ & $5988 b$ & 10,052 bc & $16.4 \mathrm{ab}$ \\
\hline RB-Cañero ( $T$ ) & $32,061 \mathrm{~b}$ & $24,184 \mathrm{c}$ & $5044 b$ & 7036 c & $9.4 \mathrm{c}$ \\
\hline CV (\%) & 22.9 & 18.5 & 24.4 & 26.1 & 12.7 \\
\hline Variedad & Bioetanol (L ha-1) & Días a floración & Altura de planta $(\mathrm{cm})$ & Acame $^{+}$ & \\
\hline Potrillo & $2026 a$ & $71.3 \mathrm{~d}$ & $233 a$ & $2.3 a$ & \\
\hline Keller $(\mathrm{T})$ & $1640 \mathrm{~b}$ & $77.6 \mathrm{c}$ & $229 a$ & $3.2 \mathrm{a}$ & \\
\hline Urja $(T)$ & $1780 a b$ & $88.3 \mathrm{a}$ & $247 a$ & $3.5 \mathrm{a}$ & \\
\hline RB-Cañero (T) & 1316 c & $82.3 b$ & $220 \mathrm{a}$ & $3.7 \mathrm{a}$ & \\
\hline CV (\%) & 23.9 & 3.7 & 8.0 & 23.7 & \\
\hline
\end{tabular}

${ }^{\dagger}$ El acame se evaluó en forma visual mediante una escala de 1 a 5, 1: sin acame y 5 muy acamada. Valores con las mismas letras para la misma variable en las columnas son estadísticamente iguales (Tukey, $\mathrm{P} \leq 0.05)$. CV: coeficiente de variación, T: testigos.

Potrillo se recomienda para siembra en las tierras bajas (0 a 800 msnm) de la región central de Nuevo León y sur de Tamaulipas. La cosecha se realiza de 20 a 25 días después de la floración, periodo en que se presenta la máxima producción de jugo y azúcar. La Facultad de Agronomía de la UANL dispone de semilla de los progenitores. Para la producción de semilla del híbrido se recomienda sembrar primero la hembra y de tres a cinco días después el macho. Este híbrido se suma al creciente número de variedades dulces disponibles en el país, que constituye un aporte genético importante para mejorar la productividad del cultivo en México.

\section{AGRADECIMIENTOS}

Se agradece el apoyo financiero proporcionado por la
Comunidad Económica Europea a través del Proyecto de Investigación No. 227422 intitulado "Sweetfuell sorghum: an alternative energy crop".

\section{BIBLIOGRAFÍA}

Murray S. C., W. L. Rooney, M. T. Hamblin, S. E. Mitchell and S. Kresovich (2009) Sweet sorghum genetic diversity and association mapping for brix and height. The Plant Genome 2:48-62, https://doi.org/10.3835/plantgenome2008.10.0011

Undersander D. J., W. E. Lueschen, L. H. Smith, A. R. Kaminski, J. D. Doll, K. A. Kelling and E. S. Oplinger (1990) Sorghum Syrup. Alternative Field Crops Manual. University of Wisconsin Cooperative Extension. Madison, Wisconsin. corn.agronomy.wisc.edu/ Crops/SorghumSyrup.aspx (August 2020).

Williams-Alanís H., F. Zavala-García, G. Arcos-Cavazos, M. C. Rodríguez-Vázquez y E. Olivares-Sáenz (2017) Características agronómicas asociadas a la producción de bioetanol en genotipos de sorgo dulce. Agronomía Mesoamericana 28:549-563, https://doi.org/10.15517/ma.v28i3.26690 